\title{
DIAGNÓSTICO DE ARRITMIAS PEDIÁTRICAS EN EL SERVICIO DE URGENCIAS: UN DESAFÍO ACADÉMICO
}

\section{DIAGNOSIS OF PEDIATRIC ARRHYTHMIAS IN THE EMERGENCY DEPARTMENT: AN ACADEMIC CHALLENGE}

\author{
Aida-Luz Giraldo ${ }^{1,6}$, GE Rueda-Merchán ${ }^{2,3,5}$, PJ Baquero- Marín ${ }^{2,3,5,7}$, DM Bados-Enríquez ${ }^{4,5,8}$.
}

\section{Señor Editor:}

Los eventos arritmogénicos en la población pediátrica, ha presentado variaciones en sus registros durante las últimas décadas con una incidencia de $5 \%$ en niños hasta de 7 años ${ }^{[1]}$. Teniendo en cuenta que ésta patología constituye un grupo complejo e infrecuente, la comunidad médica en general se ha preocupado por tener un mayor conocimiento acerca de esta entidad clínica, lo cual ha contribuido a un mayor reconocimiento de cuadros clínicos congénitos asociados a muerte súbita infantil de origen cardiaco dentro de la población pediátrica ${ }^{[2,3]}$.

En pediatría, la prevalencia de este tipo de alteraciones es menos frecuente en comparación con la población adulta. En niños, la taquicardia supraventricular, es la arritmia más frecuente, siendo la principal causa de emergencia cardiovascular en niños con un $90 \%$ de los $\operatorname{casos}^{[1]}$. La utilización de herramientas diagnósticas, como el electrocardiograma de doce derivaciones, permite valorar éste tipo de situaciones dentro del servicio de urgencias, lo cual ha disminuido el número de muertes de origen cardíaco dentro de la población infantil, sin embargo, la utilización de estas ayudas diagnósticas, no presenta un grado de utilidad superior, cuando no se tiene el grado de pericia necesario para la interpretación de los hallazgos, equipos especializados y la posibilidad de proporcionar un tratamiento oportuno ${ }^{[4]}$. Estas condiciones, conlleva a que los procesos que se llevan a cabo dentro servicio de urgencias sean limitados y que su resolución tenga que ser manejada por personal diferente al de cardiología pediátrica $^{[2,5]}$.

El reconocimiento de alteraciones del ritmo cardíaco en la población pediátrica, se ha convertido en un reto para el médico del siglo XXI, circunstancias especiales, tales como la edad del paciente y los fenómenos fisiológicos cardíacos que trae consigo el crecimiento en ésta población, genera dificultades para la interpretación de los resultados ${ }^{[6]}$. Un estudio realizado en 12 países, con más de 900 médicos de diferentes servicios, incluyendo especialistas de las diferentes áreas de medicina interna y médicos generales, concluyo que pese a tener una muestra considerable y no ser suficiente para describir toda la población médica, se encontró que menos del $50 \%$ podía reconocer plenamente las alteraciones del ritmo dentro del electrocardiograma de doce derivaciones ${ }^{[2]}$.

El uso del electrocardiograma como herramienta diagnóstica de arritmias dentro del servicio de urgencias, ha permitido que sea ampliamente valorada su utilidad en los últimos quince años, con la finalidad de ser aplicada en grupos especiales de pacientes, diferentes a los que se encuentran propiamente en este servicio, justificándose ventajas, tales como, el reconocimiento de patologías como el síndrome de QT largo, lo que podría prevenir el desarrollo de arritmias letales dentro de la edad pediátrica, y la detección de patologías cardíacas de presentación asintomática que conducen a muerte súbita infantil ${ }^{[5,6]}$.

Consideramos fundamental que la formación médica actual, siga profundizando y proporcionando las herramientas suficientes al médico general para poder detectar y abordar de manera integral las arritmias en pediatría. Además de la necesidad de tener una mayor evidencia científica que justifique la implementación del uso del electrocardiograma en pacientes con una historia clínica personal y familiar compatible con patologías que tienen relación con el síndrome de muerte súbita infantil, teniendo en

\footnotetext{
1 Departamento de Pediatría, Fundación Universitaria Autónoma de las Americas. Pereira. Colombia.

Email de contacto: hada.giraldo@gmail.com

2 Facultad de Medicina. Fundación Universitaria Autónoma de las Américas. Pereira. Colombia.

3 Semillero de Investigación en Pediatría y Puericultura (SIPEP). Fundación Universitaria Autónoma de las Américas. Pereira. Colombia.

4 Facultad de Ciencias de la Salud. Universidad del Tolima. Ibagué. Colombia.

5 Asociacion de Sociedades Científicas de Estudiantes de Medicina de Colombia (ASCEMCOL).

6 Email de contacto: hada.giraldo@gmail.com

7 Email de contacto: pedrobaquerom@gmail.com

8 Email de contacto: diegoconques@gmail.com
} 
cuenta que en nuestro país la evidencia sobre la utilidad y el uso del mismo, como mecanismo de prevención es escasa.

\section{Bibliografía}

1. David, L.B.M. and S.C.B. Esthela, Evaluación electrocardiográfica en pacientes pediátricos con arritmia cardiaca. Revista Médica de la Universidad Veracruzana, 2008. 8(2): p. 14-19.

2. Viskin, S., et al., Inaccurate electrocardiographic interpretation of long QT: the majority of physicians cannot recognize a long QT when they see one. Heart Rhythm, 2005. 2(6): p. 569-574.

3. Sanches, M., et al., Electrocardiograma en edad pediátrica. SEMERGEN-Medicina de Familia, 2014. 40(6): $p$. 334-340.

4. Saul, J.P., et al., Rationale and objectives for ECG screening in infancy. Heart Rhythm, 2014. 11(12): p. 23162321.

5. Rodday, A.M., et al., Electrocardiogram screening for disorders that cause sudden cardiac death in asymptomatic children: a meta-analysis. Pediatrics, 2012: p. peds. 2011-0643.

6. Brockmeier, K., R. Nazal, and N. Sreeram, The electrocardiogram of the neonate and infant. Journal of Electrocardiology, 2016. 49(6): p. 814-816. 